\title{
Screening success: A virtual MDT can reduce the number of patients requiring respiratory follow-up post-COVID-19 pneumonia in line with British Thoracic Society guidance
}

\author{
Authors: Rebekah Anstey, ${ }^{\mathrm{A}}$ Jennifer Rossdale, ${ }^{\mathrm{A}}$ Alexander Dereham, ${ }^{\mathrm{B}}$ Eleanor Peter, ${ }^{\mathrm{C}}$ Rey Tan, ${ }^{\mathrm{D}}$ \\ Robert Mackenzie Ross, ${ }^{\mathrm{E}}$ Graham Robinson, ${ }^{\mathrm{F}}$ Tom Hartley, ${ }^{\mathrm{E}}$ Jay Suntharalingam ${ }^{\mathrm{E}}$ and Jonathan $\mathrm{CL}$ Rodrigues ${ }^{\mathrm{G}}$
}

\section{Introduction and objectives}

The ongoing respiratory sequelae of COVID-19 pneumonia remain unclear, and the ideal follow-up of these patients is still a work in progress. We describe our experience of using a pre-follow-up multidisciplinary team (MDT) to decide the follow-up stream in patients hospitalised for COVID-19 pneumonia.

\section{Methods}

We reviewed all patients with a clinico-radiological diagnosis of COVID-19 admitted to hospital during a 3-month period and assigned a follow-up stream based on British Thoracic Society guidance.

Results

We changed the follow-up pathway in 71\% (277/392) and refined the pathway in $67 \%$ (261/392) of indeterminate cases. We also created an automated process for the general practitioner to book follow-up imaging and will use this process going forward.

Conclusion

These findings highlight the importance of the MDT review of cases with suspected COVID-19 pneumonia prior to clinic attendance to ensure appropriate patients are followed up and to optimise utilisation of outpatient imaging and clinics.

KEYWORDS: COVID-19, respiratory medicine, multidisciplinary team, ventilatory support

DOI: 10.7861/clinmed.2021-0124

Authors: ${ }^{\text {A }}$ respiratory specialist trainee, Royal United Hospital, Bath, UK; ${ }^{B}$ medical student, Bristol Medical School, Bristol, UK

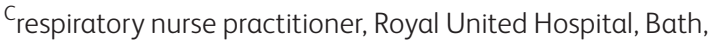
UK; ' project support officer, Royal United Hospital, Bath, UK; ${ }^{E}$ consultant of respiratory medicine, Royal United Hospital, Bath, UK; F consultant radiologist, Bristol Medical School, Bristol, UK; ${ }^{G}$ Consultant radiologist, Bristol Medical School, Bristol, UK, University of Bath, Bath, UK and Royal United Hospital, Bath, UK

\section{Introduction}

Globally we are learning to identify and manage the early-, medium- and long-term effects of COVID-19. The main anticipated chronic pulmonary sequelae are pulmonary fibrosis and pulmonary vascular disease. Pulmonary fibrosis has been seen in imaging follow-up after other coronavirus outbreaks, notably Middle East respiratory syndrome (MERS), and reported in early smaller studies during the current pandemic., ${ }^{1,2}$ Acute illness with COVID-19 appears to have high rates of venous thromboembolism. ${ }^{3,4}$ Initial studies suggested that imaging could be a marker of recovery, although further studies question its reliability in COVID-19 where patients can be quite symptomatic without any evidence of significant underlying pulmonary disease. ${ }^{5,6}$ Following the initial peak, the question arose regarding how to effectively follow up this large cohort of patients. The British Thoracic Society (BTS) guidelines provide a framework based upon disease severity but recognise that implementing their recommendations may prove difficult. ${ }^{7}$ They advise that patients with a clinico-radiological diagnosis of COVID-19 undergo specific follow-up pathways based on severity of disease. ${ }^{8}$ Generally, higher severity patients requiring intensive care or high-dependency units follow a more rapid and intensive followup (Group 1) compared with mild-moderate cases (Group 2). They suggest a multidisciplinary team (MDT) approach, utilising virtual routes, if feasible. Guidance has provided information on whom and how to follow up but have not expanded to how to efficiently set this process up. Streamlining this process is going to be vital in an already stretched service dealing with the backlog of work created by the pandemic as well as facing potential fluxes in cases as lockdown eases. Within our institution, we elected to instigate a clinico-radiological MDT prior to assigning a post-COVID-19 followup pathway (pre-FU-MDT).

We aim to assess the impact of our pre-FU-MDT for patients admitted with COVID-19 pneumonia.

\section{Methods}

Identifying patients

A comprehensive approach to identify all potential COVID-19 patients was conducted by retrospectively analysing a prospectively maintained clinical database. This was cross-referenced with coding searches, business intelligence unit 
data and radiology codes to ensure maximal patient capture. This was approved by our local audit and governance department as a clinical service evaluation project and was therefore exempt from formal ethics review or informed written consent. Microbiology records were reviewed to generate a list of all reverse transcription polymerase chain reaction COVID-19 tests performed from March 2020 to July 2020 in symptomatic patients regardless of the result (acknowledging that a clinical diagnosis of COVID-19 can occur with negative swabs owing to sensitivity of the test $71 \%$ to $98 \%$ sensitivity). ${ }^{9}$ This includes patients with potential COVID-19 infection who were not admitted or managed solely by the emergency department. These patients were then crossreferenced with a radiology search to see if a chest X-ray (CXR) had been performed (Fig 1). During the pandemic, the radiology team provided around-the-clock cover. All CXRs performed were reported in real time using British Society of Thoracic Imaging (BSTI) codes: normal (CVCX0), classic (CVCX1), indeterminate (CVCX2) or non-COVID-19 (CVCX3). ${ }^{10}$ Those with CVCX0 were excluded as there was no radiological abnormality to follow up. This comprehensive search strategy yielded a robust cohort of patients with potential clinico-radiological diagnosis of COVID-19 pneumonia eligible for follow-up.

These patients were reviewed by a respiratory nurse practitioner with consultant support and follow-up streams for patients were identified using the BTS guidelines. In total, this process identified 723 patients with a clinico-radiological diagnosis of COVID-19 requiring follow-up. The first 392 consecutive patients were allocated as shown in Table 1.

\section{Post-COVID-19 pre-follow-up multidisciplinary team}

All patients were then reviewed virtually by a pre-FU-MDT consisting of a consultant respiratory physician and a consultant

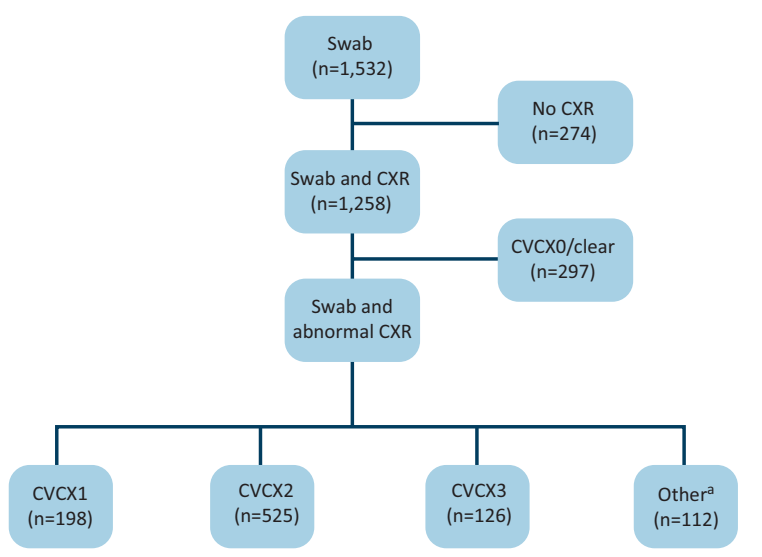

Fig 1. Reverse transcription polymerase chain reaction swabs and chest $X$-rays performed. ${ }^{a}$ Includes patients who died $(n=93)$ and paediatric patients $(n=9)$. CXR $=$ chest $X$-ray.

thoracic radiologist. There were nine meetings, lasting between 30 minutes and 2 hours. The clinician and administrative time for these meetings was re-allocated from services reduced during the pandemic. Each case was discussed using electronic patient records, results and imaging. This provided enough information to allow a clinico-radiological diagnosis.

\section{Follow-up}

Group 1: All patients for Group 1 follow-up had a standard letter sent to their general practitioner (GP), which explained that the patient had been reviewed by the MDT, clarified the diagnosis and described the plan for follow-up (supplementary material S1).

Table 1. Change in follow-up status following review at pre-follow-up multidisciplinary team

\begin{tabular}{|c|c|c|c|c|c|c|}
\hline & $\begin{array}{l}\text { Allocated to } \\
\text { Group } 1 \text { follow-up } \\
\text { without pre-FU } \\
\text { MDT }\end{array}$ & $\begin{array}{l}\text { Allocated to } \\
\text { Group } 2 \text { follow-up } \\
\text { without pre-FU } \\
\text { MDT }\end{array}$ & $\begin{array}{l}\text { Patients requiring } \\
\text { no follow-up } \\
\text { without pre-FU } \\
\text { MDT }\end{array}$ & $\begin{array}{l}\text { Non-COVID-19 } \\
6 / 52 \text { CXRs } \\
\text { without pre-FU } \\
\text { MDT }\end{array}$ & $\begin{array}{l}\text { Indeterminate } \\
\text { CXRs without } \\
\text { pre-FU MDT }\end{array}$ & Total \\
\hline $\begin{array}{l}\text { Allocated to Group } \\
1 \text { follow-up with } \\
\text { pre-FU MDT }\end{array}$ & 25 & 1 & 0 & 0 & 1 & 27 \\
\hline $\begin{array}{l}\text { Allocated to Group } \\
2 \text { follow-up with } \\
\text { pre-FU MDT }\end{array}$ & 2 & 44 & 3 & 0 & 38 & 87 \\
\hline $\begin{array}{l}\text { Patients requiring } \\
\text { no follow-up with } \\
\text { pre-FU MDT }\end{array}$ & 5 & 12 & 16 & 6 & 150 & 189 \\
\hline $\begin{array}{l}\text { Non-COVID-19 } \\
6 / 52 \text { CXRs with } \\
\text { pre-FU MDT }\end{array}$ & 3 & 8 & 1 & 1 & 65 & 78 \\
\hline $\begin{array}{l}\text { Non-COVID-19 } \\
\text { bespoke follow-up } \\
\text { with pre-FU MDT }\end{array}$ & 0 & 3 & 0 & 1 & 7 & 11 \\
\hline Total & 35 & 68 & 20 & 8 & 261 & $392^{a}$ \\
\hline
\end{tabular}




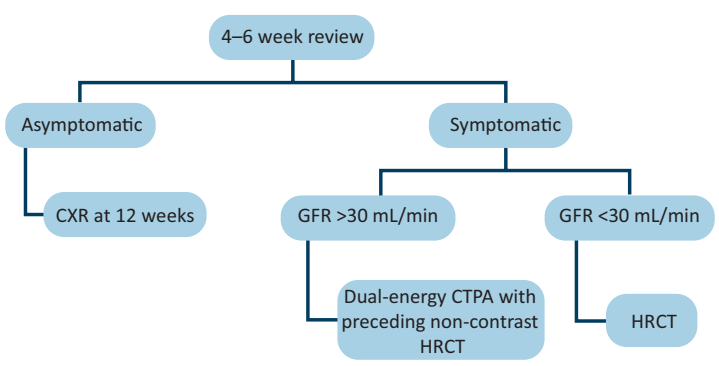

Fig 2. Algorithm for deciding on follow-up imaging in Group 1 patients. $\mathrm{CTPA}=$ computed tomography pulmonary angiography; $\mathrm{CXR}=$ chest $\mathrm{X}$-ray; GFR = glomerular filtration rate; $\mathrm{HRCT}=$ high-resolution computed tomography.

Further imaging requests in this group were delayed until the initial telephone review to ensure the correct modality was used (Fig 2).

All patients with a diagnosis of pulmonary embolic disease (PE) who were under Group 1 follow-up had their anti-coagulation managed by the respiratory team.

Group 2: Usually, it is the practice at our centre for follow-up CXRs to be organised and reviewed by GPs in the community and referred onward to specialty care if there are concerns, as per BTS guidance on pneumonia. ${ }^{11}$ Following negotiations with the clinical commissioning group, this process was extended for COVID-19 patients. The GPs were requested either by letter (supplementary material S2) or discharge summary to organise a follow-up CXR at 12 weeks. If this was abnormal or review of the patient revealed symptoms, they would be then encouraged to make an onward referral to the respiratory services. Follow-up imaging was reported as per BSTI guidance. ${ }^{10}$

\section{Outcome}

To assess the impact of the COVID-19 pre-FU MDT, we reviewed the clinical pathways of all patients discussed at the pre-FU MDT and compared with the original follow-up stream from initial allocation (based on the coded level of respiratory support and CXR codes) and assessed their clinical outcomes. Patient symptoms, pulmonary function test results and imaging were reviewed, and outcome data assessed, as suggested by BTS. Follow-up imaging was reported as per BSTI post-COVID-19 follow-up codes. ${ }^{10}$

\section{Results}

The pre-FU MDT changed the outcome or clarified the follow-up plan for a large proportion $(71 \% ; 277 / 392)$ of patients (Table 1$)$. Following discharge, $66 \%$ (86/131) of patients had no change in planned follow-up. There was a large number of patients (261) in whom discharge follow-up was unclear or not stipulated in BTS guidance. Most noticeable of these were patients with indeterminate imaging (CVCX2 or CVCT2). The pre-FU MDT allowed clarification of follow-up stream in these patients accounting for $67 \%$ (261/392) of total patients reviewed. Of these patients, $57 \%$ (150/261) had their follow-up cancelled and the remaining 43\% (111/261) were stratified to Group 1, Group 2 or other. Follow-up was ceased completely in $16 \%$ (17/108). The
pre-FU MDT changed the follow-up pathway in $21 \%$ (23/108) of patients previously allocated to Group 1 or Group 2 follow-up.

\section{Outcome data in Group 1}

Forty-one patients had been reviewed in this group at the 12-week face-to-face appointment at time of data interpretation. Sixty-one per cent (25/41) had been discharged from further follow-up. Fifty-six per cent (23/41) received invasive ventilation during inpatient admission, 27\% (11/41) received nasal high flow, one patient received continuous positive airway pressure and one bilevel positive airway pressure. The remaining 12\% (5/41) received oxygen therapy only. Two patients received extracorporeal membrane oxygenation. Imaging, CXR or computed tomography at the time of COVID-19 pneumonia showed 83\% (34/41) had 'classic' appearances (CVCX1/CVCT1) and the rest had indeterminate changes (CVCX2/CVCT2).

The majority of patients had pulmonary function testing performed at follow-up, based on symptoms at the initial contact. The average forced expiratory volume in one second $\left(\mathrm{FEV}_{1}\right)$ was $89 \%$ predicted $\mathrm{FEV}_{1}$ (range $43 \%$ to $118 \%$ ), average forced vital capacity (FVC) was $89 \%$ predicted (range $62 \%$ to $115 \%$ ). The average FEV1/FVC ratio was $81 \%$. The average diffusing lung capacity for carbon monoxide (DLCO) was $70.1 \%$ (range $40 \%$ to $111 \%$ ). A third performed a 6 -minute walk distance with an average of 402 metres (range 219 m to 672 m), with no significant desaturation. Two of these were discounted as they were stopped due to musculoskeletal issues rather than breathlessness.

On review in clinic, over half reported no limitation at the

12-week point, with two patients even commenting that they were better than before. Forty-six per cent (19/41) reported symptoms with breathlessness being the main complaint and 29\% (12/41) reported fatigue, lethargy and weakness.

All patients had repeat imaging at around 12 weeks from discharge. The majority had cross-sectional imaging. Fig 3 displays the reported outcomes. Overall, 71\% (29/41) had complete resolution or improved appearances. The patients with fibrotic changes (PCVCT3) displayed a reduced DLCO $(40 \%$ to $74 \%$ of predicted values).

There were no de novo PEs picked up on repeat imaging at 12 weeks. Thirty-nine per cent (16/41) were diagnosed with PE during admission. One of these patients was treated empirically for 3 months as they were unable to be scanned. All of these patients were commenced on anti-coagulant therapy during admission. Repeat imaging, around the 12-week point, showed resolution in $88 \%(14 / 16)$ of these patients. One patient showed evidence of right ventricular dilatation and was subsequently followed up in the pulmonary hypertension clinic, where there was no evidence of chronic thrombo-embolic pulmonary hypertension and his anticoagulation was stopped. One patient with a residual clot was continued on anticoagulation with a plan for repeat computed tomography pulmonary angiography and follow-up.

\section{Outcome data in Group 2}

One-hundred and twenty-one patients had gone through Group 2 follow-up at the time of this review. Fifty-three per cent (64/121) were CVCX1, 36\% (44/121) were CVCX2 and the rest (11\%; 13/121) were CVCX3. Ninety-two had follow-up CXRs performed. Ninety-one per cent (84/92) had improved or fully resolved appearances (PCVCX0/1). Four had died before imaging could be 


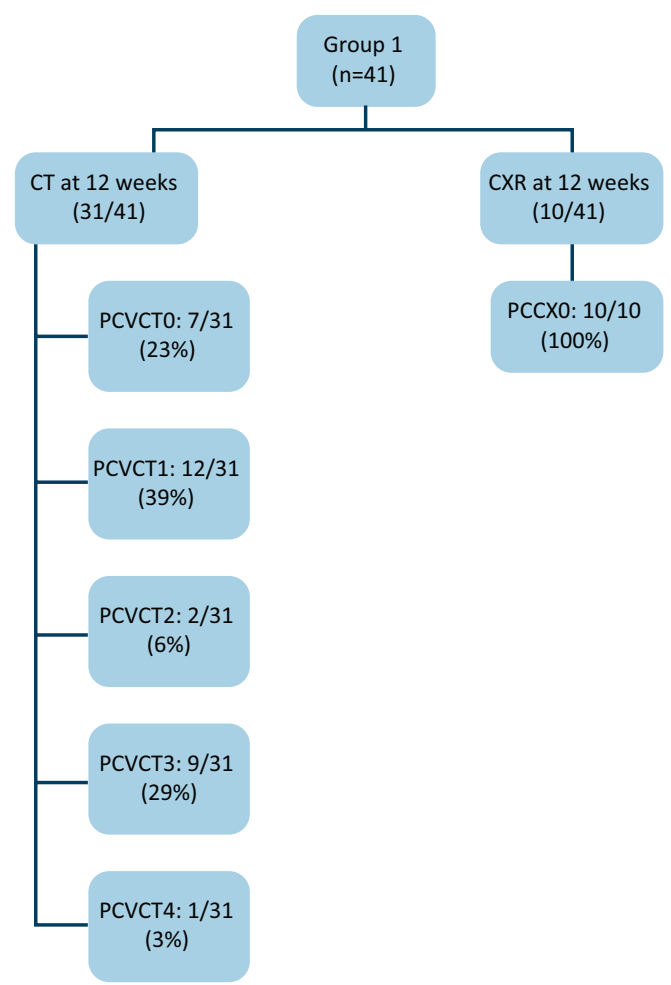

Fig 3. Imaging follow-up of Group 1. $C T=$ computed tomography; $C X R=$ chest $\mathrm{X}$-ray.

performed. Eighteen per cent (22/121) of patients had no followup CXR requested for unknown reasons. Eight per cent (10/121) had CXR follow-up requested but the patient either cancelled or did not attend follow-up. Only 4\% (5/121) of patients had abnormal or worsening imaging (PCVCX2-4) and none of these changes were felt to be related to previous COVID-19 infection. Of these, two were extremely unwell due to other diagnoses (neutropenic sepsis and disseminated fungal infection). One was already followed up by the respiratory team for an existing bronchiectasis diagnosis. Two had developed pleural effusions and were referred to the pleural clinic. Three Group 2 patients were diagnosed with PE during their COVID-19 admission and followed up by the haematology team.

\section{Discussion}

This is the first report of the impact of a pre-FU MDT for patients with clinico-radiological COVID-19 pneumonia. We demonstrate that the pre-FU MDT provides a platform to review COVID-19 follow-up pathways and reduces unnecessary follow-up in $48 \%$ of patients. The MDT has ensured clarification of a large number of indeterminate cases, which could have had unnecessary imaging or clinic followup and resultant uncertainty for the patient and primary care practitioners. It has resulted in our specialist outpatient respiratory services being able to focus on those patients most likely to benefit from specialist review. It has allowed effective redistribution of clinicians' time, in a stretched service in the midst of a pandemic.

Studies have suggested post-COVID-19 patients suffer with a significant symptom burden. ${ }^{12,13}$ Identifying which of these patients would benefit from specific respiratory follow-up for postCOVID-19 pulmonary disease is difficult. Nonetheless, follow-up data from the clinic including the low referral rate of COVID-19 patients who were allocated solely to imaging follow-up or no follow-up after pre-FU MDT (3\%; 6/189) suggest that these patients were triaged correctly. Our pickup of worsening imaging in both our follow-up groups ( 1 and 2) using this method was $9 \%$ $(14 / 162)$, similar to other studies where they performed imaging on all patients. ${ }^{12}$ Of the six patients who were referred ad hoc to the respiratory team for review, $67 \%(4 / 6)$ were diagnosed with long COVID and $33 \%$ (2/6) were diagnosed with other conditions (asthma and aortic stenosis) felt to be causing their symptoms. None of these patients required ongoing follow-up. If appropriate, these patients were referred onto community post-COVID-19 rehabilitation programmes.

Given that the NHS had been affected by the loss of staff to non-patient-facing or working from home roles, this was a good utilisation of these staff to perform initial patient identification, data collection and screening.

The improved collaboration between respiratory and radiology specialties ensured reduction in unnecessary repeated imaging and subsequent reduction in radiation dosing to individuals. This multidisciplinary teamwork has had a beneficial effect on interdepartmental communication with better advice routes.

The rapid creation of a simple-to-use tool for GPs to request follow-up CXRs aided the COVID-19 follow-up process but will also be useful in requesting follow-up imaging in the community. However, a proportion of Group 2 patients did not have repeat imaging performed. This may have been due to them being asymptomatic but there is no feedback to ascertain this. Part of the GP letter for Group 2 patients stipulates: 'If it is clinically appropriate.' Therefore, GPs may have reviewed and felt no further imaging was indicated. This is an area for future review.

Identifying and monitoring patients with abnormal imaging early will be vital in improving outcomes for the individual. Similar to previous studies, chronic post-viral symptoms were commonly reported, and holistic assessment of these patients will allow pivotal signposting to other services and rehabilitation. We can use the outcome data from these patients to anticipate local service needs.

This process also led to establishing standard operating procedures to follow up COVID-19 patients. Patients admitted to hospital now are prospectively allocated to the correct follow-up groups. Hospital-wide briefings, grand round events and ad hoc education has disseminated this message. The respiratory team actively identify and triage 'Group 1 patients' and appointments generated by a single administrator. CXR reports offer standardised advice for the follow-up of group 2 patients to ensure ongoing re-enforcement.

\section{Limitations}

We understand that this method has worked well in our trust, however, may not be applicable or feasible in all centres. We hope that others can use our pre-FU MDT as a template. Our Group 2 follow-up relies heavily on our electronic patient record requesting system and the local GPs to arrange follow-up imaging. We recognise that a number of our Group 2 patients did not receive any follow-up imaging and, for most of these, the reason is unknown. Given the good outcomes in imaging and low referral rates in Group 2 patients, it is likely these patients have 
had ongoing good recovery and have had interaction with their GP negating the need for follow-up imaging. However, without pro-actively following up these patients, we will not be certain. For this reason, on discharge, Group 2 patients should be advised to seek medical advice if they have ongoing or worsening respiratory symptoms.

We appreciate this system may not be achievable for all. We also recognise that our initial patient capture method of crossreferencing imaging with swab results is not applicable now as all patients are swabbed on admission to hospital not just symptomatic patients. In addition, there may be a small number of patients who were positive or symptomatic for COVID-19 who did not have a CXR on admission.

We appreciate that by focusing solely on imaging follow-up in Group 2 patients, we may miss patients who are symptomatic, although we have only received six re-referrals in this category to date. On review of literature of follow-up imaging in severe COVID-19, it is suggested that it does not necessarily correlate with symptoms. ${ }^{6}$ Fortunately, all the severe COVID-19 patients received telephone and face-to-face review of symptoms as well as imaging to address this. Not all patients had standardised pulmonary function testing on follow-up. This was due to different test requests by individual physicians, difference in patient needs and abilities, as well as limitations on our physiology laboratory due to changed protocols with the additional time needed for aerosol generating procedures. This is something we could address with ongoing follow-up planning.

\section{Conclusion}

Overall, our department has benefited from the effective redistribution of clinicians' time and use of non-patient-facing staff to reduce follow-up imaging as well as clinic appointment

\section{Summary}

\section{What is known?}

The ongoing respiratory sequelae of COVID-19 pneumonia remain unclear, and the ideal follow-up of these patients is still a work in progress.

\section{What is the question?}

How do we effectively and correctly follow up these patients to identify long-term pulmonary sequelae? How do we ensure national standards are met in follow-up in an already stretched service dealing with the fallout from a global pandemic?

\section{What was found?}

We created a pre-follow-up multidisciplinary team (MDT) to decide the follow-up stream in patients hospitalised for COVID-19 pneumonia, where we reviewed all patients with a clinicoradiological diagnosis of COVID-19 admitted to hospital during a 3-month period and assigned the follow-up stream based on British Thoracic Society guidance.

\section{What is the implication for practice now?}

We changed the follow-up pathway in $71 \%$ (277/392) and refined the pathway in $67 \%$ (261/392) of indeterminate cases. Overall highlighting the importance of the MDT review of cases with suspected COVID-19 pneumonia prior to clinic attendance to ensure appropriate patients are followed up and to optimise utilisation of outpatient imaging and clinics. time. We changed the follow-up in $48 \%$ of cases, releasing significant outpatient capacity while maintaining standards to allow early identification and follow-up of those who will most benefit. Our suggestion would be for other centres to use a similar process to improve patient care and build robust, safe patient follow-up mechanisms as we deal with COVID-19 patients requiring follow-up review and imaging. Only time will tell what the long-term effects on individual patients will be but, by using this tool, we can effectively identify the patients early that may well need continued support or treatments in the future.

\section{Supplementary material}

Additional supplementary material may be found in the online version of this article at www.rcpjournals.org/clinmedicine:

S1 - Group 1 standard follow-up letter sent to their GP.

S2 - Group 2 request letter sent to their GP.

\section{Acknowledgements}

We would like to thank Noeleen Foley, Clare Marchand, Vidan Masani, Rebecca Mason, Sharon Sturney, Toby Hall, Ben Hudson, Alison Sandeman, Caroline Styles, Rich Wood and Katy Lomas.

\section{Conflicts of interest}

Jonathan CL Rodrigues declares consultancy fees from NHSX and speaker's fees from Sanofi.

Jay Suntharalingam declares consultancy and speaker's fees from Janssen Pharmaceuticals, MSD, AstraZeneca, Chiesi, GSK and Acceleron Pharma.

\section{References}

1 Das KM, Lee EY, Singh R et al. Follow-up chest radiographic findings in patients with MERS-CoV after recovery. Indian J Radiol Imaging 2017;27:342-9.

2 Han X, Fan Y, Alwalid O et al. Six-month follow-up Chest CT Findings after Severe COVID-19 Pneumonia. Radiology 2021;299:E177-86.

3 Giannis D, Ziogas IA, Gianni P. Coagulation disorders in coronavirus infected patients: COVID-19, SARS-CoV-1, MERS-CoV and lessons from the past. J Clin Virol 2020;127:104362.

4 British Thoracic Society. BTS guidance on venous thromboembolic disease in patients with COVID-19. BTS, 2020.

5 Rousan LA, Elobeid E, Karrar $M$ et al. Chest $x$-ray findings and temporal lung changes in patients with COVID-19 pneumonia. BMC Pulm Med 2020;20:245.

6 D'Cruz R, Waller MD, Perrin F et al. Chest radiography is a poor predictor of respiratory symptoms and functional impairment in survivors of severe COVID-19 pneumonia. ERJ Open Research 2021;7:00655-2020.

7 British Thoracic Society. British Thoracic Society guidance on respiratory follow-up of patients with a clinico-radiological diagnosis of COVID-19 pneumonia. BTS, 2020.

8 George PM, Barratt SL, Condliffe R et al. Respiratory follow-up of patients with COVID-19 pneumonia. Thorax 2020;75:1009-16.

9 Watson J, Whiting PF, Bush JE. Interpreting a covid-19 test result. BMJ 2020;369:1808.

10 British Society of Thoracic Medicine. BSTI COVID-19 followup CT and CXR report codes. BSTI, 2020. www.bsti.org.uk/covid-19resources/covid-19-bsti-reporting-templates

11 British Thoracic Society. Guidelines for management of community acquired pneumonia in adults. Thorax 2009;64 (supp III):iii1-55. 
12 Mandal S, Barnett J, Brill SE et al. 'Long-COVID': a cross-sectional study of persisting symptoms, biomarker and imaging abnormalities following hospitalisation for COVID-19. Thorax 2021;76:396-8.

13 Carfi A, Bernabei R, Landi F. Persistent symptoms in patients after acute COVID-19. JAMA 2020;324:603-5.
Address for correspondence: Dr Jonathan Rodrigues,

Department of Radiology, Royal United Hospitals Bath NHS

Foundation Trust, Combe Park, Bath BA1 3NG, UK.

Email: j.rodrigues1@nhs.net

Twitter: @JCLRodrigues 